\title{
Que reste-t-il comme indications au traitement chirurgical non prothétique de la coxarthrose
}

\author{
R. Petit \\ 16, avenue Clemenceau, F-68100 Mulhouse
}

Sous ce titre prétentieux se dissimule une interrogation presque quotidienne pour tout chirurgien orthopédiste, devant certains patients adressés pour la réalisation d'une arthroplastie par prothèse totale. Souvent convaincus par des opérés, ils ont fait leur choix, présentent une coxopathie évoluée et n'envisagent guère d'alternative thérapeutique. Certains ont déjà un dossier radiologique étoffé dans lequel l'on découvre que, quelques années plus tôt, une intervention palliative aurait été très efficace. Parfois le jeune âge du patient, les données radiologiques incitent à approfondir quand même le bilan et à dégager une indication d'ostéotomie qui n'apporte qu'un soulagement relatif. La conviction d'être à l'abri des complications de l'arthroplastie fait bientôt place à l'amertume chez bon nombre de ces rescapés de la prothèse totale qui, quelques années plus tard, sont réopérés. Cette prothèse qu'ils revendiquent est d'autant plus légitime que les bons résultats fonctionnels sont devenus réguliers, et que la stabilité des résultats dans le temps s'est indéniablement améliorée.

Ces considérations expliquent la diminution générale de la fréquence des ostéotomies, flagrante dans l'activité des membres du GECO qui ont tous été habitués, au cours de leur formation, à maîtriser les indications et la réalisation technique des ostéotomies, qui en ont une solide expérience, et qui restent soucieux de préserver le capital osseux.

La connaissance de la biomécanique est indispensable pour discuter valable- ment les indications d'ostéotomies, mais elle n'est pas suffisante pour garantir le résultat fonctionnel, confirmant que la chirurgie osseuse n'est pas une science mais un art. Comprendre ce que l'on crée est de toute évidence un devoir impératif. L'approche très didactique de Pauwels, dont chacun retient le concept mécanique de la hanche dans le plan frontal, est utilement complétée par les travaux de Teinturier pour envisager l'aspect tridimensionnel de la hanche. La conception élaborée de la hanche arthrosique apportée par Bombelli et le schéma dynamique de Frain affinent la représentation mécanique de la hanche et doivent être prises en compte dans la réflexion de chaque indication. Toutes les modifications apportées à la hanche retentissent sur les articulations sous-jacentes, la hanche controlatérale et le rachis. La planification de l'intervention représente, on le voit, un travail bien plus élaboré que la réalisation technique de l'intervention. Ces considérations valent aussi pour l'arthroplastie par prothèse totale dont la planification est incontestablement facilitée par le caractère plus prévisible de la qualité de l'interface articulaire et de l'architecture coxofémorales post-opératoires.

La qualité du bilan radiologique pré-opératoire a donc une importance primordiale. Nous considérons comme indispensables une radiographie du bassin de face en charge, le cliché de faux profil et le profil standard, les clichés de recentrage en abduction et en adduction. Un cliché de recentrage en flexion et en extension de profil est utile chaque fois que l'articulation n'est plus concentrique sur l'incidence de profil. Nous ne développons pas ce chapitre, car nous n'avons pas d'élément original à apporter. Nous soulignons simplement que nous n'utilisons jamais de cliché de recentrage sous anesthésie. Il faut enfin préciser que bien que ce bilan ne renseigne pas de façon satisfaisante sur l'état du cartilage, nous n'avons jamais exploité les renseignements apportés par l'arthrographie opaque.

La série de 168 interventions de chirurgie palliative que nous présentons n'est homogène que par l'existence de lésions d'arthrose confirmée. Nous avons voulu évaluer l'efficacité du traitement chirurgical non prothétique de la coxarthrose et ne prenons pas en compte la chirurgie de prévention.

Cette série de 140 patients, dont 28 ont bénéficié d'une intervention bilatérale, comprend $60 \%$ de femmes et $40 \%$ d'hommes. Les femmes ont en moyenne 43 ans au moment de l'intervention et présentent en majorité une coxarthrose secondaire, alors que les hommes sont en moyenne plus âgés, 47 ans, et présentent une plus grande fréquence de coxarthroses primitives. La douleur représente le motif significatif de l'intervention, les hanches raides n'ayant guère de chances d'être améliorées par la majorité des techniques présentées.

Il existe, dans la majorité des cas, une corrélation entre la cotation clinique et la cotation radiologique.

Regroupant l'expérience de plus de 
dix chirurgiens différents, cette étude rétrospective ne permet pas d'établir des statistiques significatives, mais tout au plus de dégager prudemment des tendances. La plus évidente de ces tendances est la raréfaction des interventions palliatives dans la chirurgie de la coxarthrose depuis 1985, opposée à une augmentation de fréquence des prothèses totales de hanche.

La butée extra-articulaire (14 hanches) permet de stabiliser des lésions arthrosiques peu évoluées dues à une dysplasie acétabulaire isolée. L'association d'une coxa valga, et a fortiori d'un décrochage même minime du cintre cervico-obturateur contre-indiquent cette technique car elle ne fait qu'augmenter la surface de transmission des contraintes sans en modifier la direction.

L'ostéotomie de Chiari (10 hanches) combine l'effet de couverture de la tête fémorale à la modification de la direction et de l'importance des contraintes et permet de stabiliser des lésions plus avancées. Sa technique est plus exigeante et les suites opératoires sont plus lourdes.

L'ostéotomie fémorale de varisation (81 hanches) est la plus utilisée. Elle combine la réduction des contraintes par détente musculaire, la modification de leur direction, et l'augmentation de la surface de leur répartition. L'association d'une translation diaphysaire interne (28 cas) n'améliore pas la qualité des résultats dans notre série. Indiquée dans la coxarthrose primitive aussi bien que dans les coxarthroses sur dysplasie, elle ne doit être retenue que lorsque le cliché de recentrage en abduction montre non seulement une bonne congruence mais surtout un bon interligne. C'est avant 50 ans que les résultats de notre série sont les meilleurs, indépendamment du facteur surcharge pondérale que l'on considère communément comme néfaste.

Isolée, l'ostéotomie de translation diaphysaire interne dite de McMurray (19 hanches) donne des résultats décevants. Résolument antalgique par son rôle primordial de détente musculaire, elle n'a procuré un soulagement durable que chez 5 patients $(26 \%)$.
L'ostéotomie de flexion céphalique (6 hanches) nous a déçus, et n'est plus utilisée isolément. Rétablissant la congruence sagittale de la hanche, elle détend les fléchisseurs en avançant leur insertion distale. Le résultat antalgique est remarquable mais de brève durée dans notre courte série.

L'ostéotomie de valgisation (21 hanches) recherche une augmentation de la surface portante et une modification de la direction des contraintes. Elle ne donne de résultats vraiment satisfaisants que dans les arthroses sur dysplasie mixte présentant un ostéophyte « en goutte », ce qui correspond à l'indication la plus unanimement reconnue.

Les ostéotomies combinées de Bombelli représentent la seule série homogène : elle émane d'un seul service, et les indications ont été posées en collaboration avec l'auteur de la méthode. Elles ne sont pas intégrées dans notre statistique générale. Inspirées par le souci de réharmoniser et non de normaliser l'équilibre des forces agissant sur la hanche, elles donnent des résultats intéressants.

Satisfaire à ces principes biomécaniques et techniques ne saurait masquer la réalité clinique faite, certes, de succès chirurgical - car les complications de ces interventions sont rares - mais aussi de la combativité des patients. La rééducation post-opératoire est dans bien des cas symbolique, se limitant à encadrer et parfois endiguer les efforts de patients déterminés parmi lesquels l'on retrouve le plus fort pourcentage de bons résultats. Elle devient problématique pour beaucoup de patients d'âge volontiers élevé au contexte socio-familial et professionnel souvent perturbé, chez lesquels une véritable prise en charge est nécessaire, avec ses contraintes d'hébergement et de financement. Le résultat final de ces derniers patients est fréquemment insuffisant, et parfois paradoxal par rapport à nos évaluations chiffrées.

L'échec contraint à la reprise chirurgicale. Nous n'avons pas particulièrement étudié les « ré-ostéotomies » qui traduisent plus souvent l'imperfection technique d'une intervention que les erreurs dans le choix d'une méthode. Les reprises par prothèses totales concrétisent l'échec complet (25 cas) pour les 9 hanches reprises avant la deuxième année post-opératoire et les 16 hanches réopérées avant la cinquième année post-opératoire, et l'échec plus relatif pour les 10 autres reprises réalisées avec un recul de cinq à sept ans. A ces trois périodes distinctes de reprises correspondent des difficultés techniques certes surmontables et décroissantes avec le temps. Seules les fortes translations internes et surtout les angulations sagittales (ostéotomies de flexion) soulèvent parfois des problèmes délicats.

L'analyse des résultats obtenus est guidée par le souci d'évaluer l'efficacité réelle du traitement palliatif réussi, en éliminant de rares dossiers d'arthrose jugée débutante, et en éliminant les échecs supposés techniques, donc toutes les reprises. Considérées globalement, toutes ces techniques entraînent au cours des deux premières années postopératoires une amélioration de la cotation de la douleur, mais ce bénéfice s'épuise dans le temps pour devenir faible sept ans après l'intervention. La mobilité, en revanche, est peu améliorée dans les deux premières années et devient inférieure à la cotation préopératoire vers la septième année. La marche suit la cotation de la douleur avec un décalage vers le bas. Seules $64,2 \%$ des hanches opérées sont faiblement améliorées sept ans après l'intervention et l'on relève $65,7 \%$ d'aggravations radiologiques.

La comparaison de ces résultats à ceux de la série de prothèses totales sans ciment présentée par le GECO en janvier 1989, concernant des implants actuellement abandonnés au profit d'implants non cimentés nettement plus performants, montre de bien meilleurs résultats cliniques pour l'arthroplastie sans ciment.

Malgré cette comparaison, le traitement chirurgical non prothétique de la coxarthrose établie conserve bien entendu des indications nombreuses, mais leur sélection doit être plus sévère et plus exigeante. Les coxarthroses sur dysplasie en constituent le plus gros 
contingent. La coxarthrose primitive reste accessible aux ostéotomies, chez des patients coopérants et devant des lésions bien sélectionnées. L'on peut regretter que les indications les moins contestables d'ostéotomies concernent des souffrances cliniques bien calmées par la riche pharmacopée des anti- inflammatoires, et que l'avis du chirurgien soit rarement sollicité, à ce stade réputé à tort relever du traitement médical.

Il y a quinze ans, l'enseignement universitaire et post-universitaire accordait une place importante au traitement palliatif de la coxarthrose et surtout au traitement préventif des dysplasies. La raréfaction de cet enseignement au profit de celui des succès de l'arthroplastie explique partiellement la régression systématique et préoccupante de la fréquence des ostéotomies les moins contestables dans notre activité chirurgicale. 